\title{
John Tsiouris, Pina C. Sanelli, Joseph P. Comunale: Case-Based Brain Imaging, 2nd Edition
}

\author{
Thieme Medical Publishers, New York, 2013; ISBN 978-1-60406-953-2
}

\author{
Antonio Boemio • Luigi Mansi
}

Published online: 6 December 2013

(C) Springer-Verlag Berlin Heidelberg 2013

This book is the second edition of the popular Teaching Atlas of Brain Imaging by Fischbein, Dillon and Barkovich, published about 12 years ago. It is completely revised with respect to the first edition, being based on the latest technological advances in brain imaging. The current editors are A. John Tsiouris, Associate Professor of Clinical Radiology, Pina C. Sanelli, Associate Professor of Radiology and Public Health, and Joseph P. Comunale, Associate Professor of Clinical Radiology, all working at the Weill Cornell Medical College in New York. The foreword was written by Robert D. Zimmermann, Professor of Radiology and Vice Chair for Education in the same institution. The authors have combined their clinical and didactic expertise to produce a useful, concise and easily readable text that explains the primary role of neuroimaging in a broad spectrum of diseases of the nervous system.

The book consists of 678 pages describing more than 150 clinical cases divided into seven sections, each section taking into account pathologies of the nervous system. The reader is presented with an interesting selection of example images of: (1) supratentorial and infratentorial neoplasms, (2) infectious and noninfectious inflammatory diseases, (3) cerebrovascular diseases , (4) neurodegenerative/white matter/ metabolic diseases, (5) trauma, (6) congenital/developmental malformations and syndromes, including supratentorial and infratentorial, malformations of cortical development and phakomatoses, and (7) cranial nerves. Each case study consists of a short clinical presentation and of several radiological images with an accurate description of the alterations shown. In this way the diagnosis is reached through a wide analysis of the differential diagnoses based on a detailed evaluation of the disease in question. To reach this didactic goal, risk factors, clinical and radiological findings, aetiology and complications are widely discussed; many rare "zebra" cases that can cause diagnostic dilemmas, and are thus a source of medical errors, are also included.

The highest value of the book is in the more than 1,000 updated high-resolution images acquired using state-of-the-art equipment. This series is further enriched by the possibility of a database of more than 250 clinical cases studied with neuroimaging accessible online, that would allow fast and practical reference to some of the most common and/or critical diseases of the nervous system evaluated through their radiological changes.

In our opinion, the book may be considered as a guide of exceptional value in the diagnosis and management of patients with a pathology of the nervous system. Therefore, it may represent a useful reference manual for specialists and radiologists in training. In particular it could serve as an excellent review for radiology oral board examinations or the Certificate of Added Qualification examination. The presence of many clear examples of the most common diseases of the nervous system would be of interest even to neurologists and neurosurgeons, since neuroimaging is an indispensable component of their activities for the diagnosis and treatment of brain disorders.

\footnotetext{
A. Boemio $\cdot$ L. Mansi $(\square)$

Second University of Naples, Naples, Italy

e-mail: luigi.mansi@unina2.it 